PROCEEDINGS OF THE

AMERICAN MATHEMATICAL SOCIETY

Volume 126, Number 3, March 1998, Pages 745-750

S 0002-9939(98)04087-8

\title{
UNIQUENESS FOR AN OVERDETERMINED BOUNDARY VALUE PROBLEM FOR THE P-LAPLACIAN
}

\author{
FARID BAHRAMI AND HENRIK SHAHGHOLIAN
}

(Communicated by J. Marshall Ash)

\begin{abstract}
For $p>1$ set $\Delta_{p} u=\operatorname{div}\left(|\nabla u|^{p-2} \nabla u\right)$, and let $\mu$ be a measure with compact support. Suppose, for $j=1,2$, there are functions $u_{j} \in W^{1, p}$ and (bounded) domains $\Omega_{j}$, both containing the support of $\mu$ with the property that $\Delta_{p} u_{j}=\chi_{\Omega_{j}}-\mu$ in $\mathbf{R}^{N}$ (weakly) and $u_{j}=0$ in the complement of $\Omega_{j}$. If in addition $\Omega_{1} \cap \Omega_{2}$ is convex, then $\Omega_{1} \equiv \Omega_{2}$ and $u_{1} \equiv u_{2}$.
\end{abstract}

\section{INTRODUCTION}

The inverse "exterior" domain problem in classical potential theory is concerned with finding the shape of a domain, given its Newtonian potential at the exterior points. One of the main features of this problem is to give geometric conditions so that there is at most one domain having prescribed exterior potential.

A method for approaching this problem is to assume the contrary, i.e. to assume that there are, at least, two solutions $\Omega_{j}(j=1,2)$, whose Newtonian potentials

$$
U^{\Omega_{j}}(x)=c_{N} \int_{\Omega_{j}} \Gamma(x-y) d y \quad(j=1,2)
$$

coincide outside their union. Here

$$
\Gamma(x)= \begin{cases}|x|^{2-N}, & N \geq 3, \\ \log |x|, & N=2,\end{cases}
$$

and $c_{N}$ is a normalization factor so that $\Delta U^{\Omega_{j}}=\chi_{\Omega_{j}}$ in $\mathbf{R}^{N}$. Next, one defines

$$
U=\left\{\begin{array}{lll}
U^{\Omega_{1}} & \text { in } & \Omega_{1}^{c}, \\
U^{\Omega_{2}} & \text { in } & \Omega_{2}^{c}, \\
\text { arbitrary } & \text { in } & \Omega_{1} \cap \Omega_{2} .
\end{array}\right.
$$

Since $U^{\Omega_{1}}=U^{\Omega_{2}}$ in $\left(\Omega_{1} \cup \Omega_{2}\right)^{c}, U$ is well defined. One may also assume $U$ is $C^{1}$ in a small neighborhood of any point $x \in\left(\partial \Omega_{1} \cap \Omega_{2}\right) \cup\left(\partial \Omega_{2} \cap \Omega_{1}\right)$. This depends on the fact that the Newtonian potential, with bounded density, is $C^{1}\left(\mathbf{R}^{N}\right)$.

Now setting

$$
u_{j}=U^{\Omega_{j}}-U, \quad \text { in } \mathbf{R}^{N} \quad(j=1,2)
$$

Received by the editors April 3, 1996 and, in revised form, August 28, 1996.

1991 Mathematics Subject Classification. Primary 31B20, 35J05, 35R35.

Key words and phrases. Inverse domain problem, p-Laplacian, uniqueness.

(C)1998 American Mathematical Society 
we'll have, for $j=1,2$,

$$
\begin{aligned}
& \Delta u_{j}=\chi_{\Omega_{j}}-\mu \quad \text { in } \mathbf{R}^{N}, \\
& u_{j}=0 \quad \text { in } \mathbf{R}^{N} \backslash \Omega_{j},
\end{aligned}
$$

where $\mu=\Delta U$, with

$$
\operatorname{supp} \mu \subset\left(\Omega_{1} \cap \Omega_{2}\right) \cup\left(\partial \Omega_{1} \cap \partial \Omega_{2}\right) .
$$

We also remark that $\Omega_{1} \cap \Omega_{2} \neq \emptyset$, since otherwise $U^{\Omega_{j}}$ can be continued, harmonically, into $\Omega_{j}$ and violate Liouville's theorem, unless $\Omega_{j}=\emptyset$, which is excluded.

The above described problem, through equation (1), now becomes a "free boundary problem", which has been studied by several people (see [sa], [sh], [gu], [g-s], [h-k-m]). For the original problem of uniqueness in the class of domains with the same exterior potentials we refer to the book of Isakov [i] and the references therein, (see also $[\mathrm{z}]$ ).

A famous result of P.S. Novikov [n] states that uniqueness holds in the class of starshaped domains with the same exterior potentials. It was also conjectured that if $\Omega_{1}$ is convex, $\Omega_{2}$ is solid (no holes) and they have the same exterior potentials in the complement of their union, then they must be identical. This is still an open problem. The second author, however, proved the following partial result [shah]: If two domains, whose intersection is convex, have the same exterior potentials in the complement of their union, then they must be identical.

A generalization of the above concept to nonlinear potential theory seems not so easy, as there are no integral representations for nonlinear operators in general. However, the reformulation of the problem of potentials to the language of free boundaries (see equation (1)) seems to be a possible way of extending the concept in mind to nonlinear operators. Now the problem becomes to prove uniqueness, under certain geometric conditions, for the solutions to

$$
\begin{cases}A(u)=\chi_{\Omega}-\mu & \text { in } \mathbf{R}^{N}, \\ u=0 & \text { in } \mathbf{R}^{N} \backslash \Omega, \\ \operatorname{supp} \mu \subset \Omega, & \end{cases}
$$

where $\mu$ is a given measure and $A$ is a general operator.

In this note we will be concerned with the p-Laplace operator $\Delta_{p}$, where $1<$ $p<\infty$ and

$$
\Delta_{p} u=\operatorname{div}\left(|\nabla u|^{p-2} \nabla u\right) .
$$

By a solution to (3) when $A=\Delta_{p}$ we mean a function $u \in W^{1, p}(\Omega)$ such that

$$
\int_{\mathbf{R}^{N}}|\nabla u|^{p-2} \nabla u \cdot \nabla \phi d x=\int_{\mathbf{R}^{N}} \phi \chi_{\Omega}(d x-d \mu),
$$

whenever $\phi \in C_{o}^{\infty}\left(\mathbf{R}^{N}\right)$. Both existence and regularity of the solutions to equation (3), when $A$ is the ordinary Laplacian $(p=2$ in (4)), have been intensively studied (see [sa], [gu], [g-s], [k-s]). Most of those results may easily be generalized to hold for the p-Laplacian.

We also remark that outside the support of the measure $\mu$, any solution $u$ to equation (3) (with $A=\Delta_{p}$ ) is $C^{1, \alpha}$, which is the maximum regularity that one should expect [1]. Therefore we will always assume that our solutions are $C^{1, \alpha}$ outside the support of the measure and that they are in $W^{1, p}$ in the entire Euclidean space. The only point where we use the $C^{1}$ regularity is in connection with a Hopf's type maximum principle. 
The main result of this paper states that if $u_{1}$ and $u_{2}$ are solutions to equation (3) with supports $\Omega_{1}$ and $\Omega_{2}$ respectively and $A$ being the p-Laplacian, then $u_{1}=u_{2}$, provided $\Omega_{1} \cap \Omega_{2}$ is convex. We prove this by contradiction using the comparison and Hopf's maximum principle. For the reader's convenience we state the comparison principle (cf. ([h-k-m, 6.5, 7.6]) and [l]).

Comparison Principle 1. Let $G$ be a bounded subset of $\mathbf{R}^{N}$ and $u, v$ be two functions in $W^{1, p}(G)$ satisfying $\Delta_{p} u \geq \Delta_{p} v$ (weakly) in $G$ and $\lim \sup _{y \rightarrow x} u \leq$ $\liminf _{y \rightarrow x} v$, where $x \in \partial G$. Then $u \leq v$ in $G$.

\section{Main Result}

Theorem 1. Let $\mu$ be a measure with compact support and suppose $u_{j} \in W^{1, p}\left(\mathbf{R}^{N}\right)$ $(j=1,2)$ are solutions to

$$
\begin{cases}\Delta_{p} u_{j}=\chi_{\Omega_{j}}-\mu & \text { in } \mathbf{R}^{N}, \\ u_{j}=0 & \text { in } \mathbf{R}^{N} \backslash \Omega_{j}, \\ \operatorname{supp} \mu \subset \Omega_{j}, & \end{cases}
$$

where $\Omega_{j}$ is compact. Suppose also $\Omega_{1} \cap \Omega_{2}$ is convex. Then $\Omega_{1} \equiv \Omega_{2}$, and $u_{1} \equiv u_{2}$.

In order to prove this theorem we need some lemmas. First we prove a simple form of Hopf type maximum principle (which is also called the Hopf's boundary point lemma). However, the reader should be aware that the usual Hopf's boundary point lemma is also valid for non-degenerate elliptic operators (see $[\mathrm{t}]$ ).

Lemma 2. Let $B_{r}$ be a ball of radius $r$. Let $u \in C^{1}\left(\bar{B}_{r}\right) \cap W^{1, p}(B)$ satisfy $u \leq 0$ and $\Delta_{p} u \geq \alpha$, for some $\alpha>0$. Suppose also there is a point $z \in \partial B_{r}$ such that $u(z)=0$. Then $\frac{\partial u}{\partial n}(z)>0$, where $n$ is the unit outward normal on $\partial B_{r}$.

Proof. Consider the function $h(x)=c\left(|x|^{q}-r^{q}\right)$, where $q$ is the conjugate of $p$, i.e. $1 / p+1 / q=1$, and $c>0$ is chosen such that $\Delta_{p} h=\alpha$. Thus in $B_{r}, \Delta_{p} u \geq \Delta_{p} h$, while on $\partial B_{r}, h \geq u$. Hence by comparison principle $h \geq u$ in $B_{r}$. Since $h(z)=$ $u(z)=0$, we obtain

$$
\frac{\partial u}{\partial n}(z) \geq \frac{\partial h}{\partial n}(z)=c q r^{q-1}>0 .
$$

The above lemma holds true for $\alpha=0$. However, in the proof one should take the fundamental solution of the p-Laplacian as an auxiliary function.

Lemma 3. Let $u$ be a solution of (5) and $\Omega$ the corresponding domain. Then for each boundary point $z \in \partial \Omega$ there is a sequence $\left\{z^{j}\right\} \subset \Omega$, such that $z^{j} \rightarrow z$ and $u\left(z^{j}\right)>0$.

Proof. Let $z \in \partial \Omega$ be fixed and suppose on the contrary that there is a small ball $B$ centered at $z$ such that $u \leq 0$ on $B$. According to (5), $u$ is a subsolution in $B$ and therefore, by the strong maximum principle [h-k-m, 6.5], it cannot attain its maximum in the interior of $B$. Since $u(z)=0$, we obtain a contradiction, and the proof is completed.

Lemma 4. Under the assumptions of Theorem 1,

$$
\sup _{\Omega_{1} \backslash \Omega_{2}} u_{1} \geq \frac{1}{q} d_{1}^{q}
$$

where $d_{1}=\sup _{z \in \Omega_{1}} \operatorname{dist}\left(z, \Omega_{1} \cap \Omega_{2}\right)$. The same conclusion holds for $u_{2}$, mutatis mutandis. 
Proof. Let $z \in \Omega_{1} \backslash \Omega_{2}$ and $u(z)>0$. Let $y \in \partial\left(\Omega_{1} \cap \Omega_{2}\right)$ be the nearest point to $z$. By translation and rotation we may assume $y$ is the origin and $z$ is on the positive $x_{1}$ axis. Now let us denote by $D$ the domain $\left\{x \in \Omega_{1} ; x_{1}>0\right\}$. Set $v(x)=1 / q\left|x_{1}-z_{1}\right|^{q}$, and observe that $\Delta_{p} v=1$ and $v(x)>0>u_{1}(x)-u_{1}(z) / 2$ on $\partial D \backslash\left\{x_{1}=0\right\}$. If also $v(x) \geq u_{1}(x)-u_{1}(z) / 2$ on $\left\{x_{1}=0\right\}$, then by the comparison principle $v \geq u_{1}-u_{1}(z) / 2$ in $D$. This contradicts the simple fact that $v(z)=0<u_{1}(z) / 2$. Therefore we conclude that

$$
v \leq \sup _{\left\{x_{1}=0\right\}} u_{1}-u_{1}(z) / 2 \leq \sup _{\Omega_{1} \backslash \Omega_{2}} u_{1}-u_{1}(z) / 2 .
$$

By Lemma 3, for each boundary point $z \in \partial \Omega_{1} \backslash \bar{\Omega}_{2}$ one may consider a sequence $z^{j} \rightarrow z$ and such that $u\left(z^{j}\right)>0$. Hence (6) holds also for all boundary points $z$ off $\bar{\Omega}_{2}$. Taking the supremum for $v$ over $z$ yields the desired result.

Lemma 5. Under the hypotheses of Theorem 1 and Lemma 4 , if $\Omega_{1} \neq \Omega_{2}$ and $d_{1} \leq d_{2}$, then there exists $y^{1} \in \Omega_{1} \backslash \Omega_{2}$ such that $-u_{1}\left(y^{1}\right)>d_{1}^{q} / q$, and $\left|\nabla u_{1}\left(y^{1}\right)\right|=0$. Similar conclusions hold if $d_{2} \leq d_{1}$, mutatis mutandis.

Proof. Let $a=-\inf _{\Omega_{1} \backslash \Omega_{2}} u_{1} \geq 0$ and $y^{1} \in \bar{\Omega}_{1} \backslash \Omega_{2}$ be such that $u_{1}\left(y^{1}\right)=-a$. Then as measures

$$
\Delta_{p}\left(u_{1}+a\right) \leq \Delta_{p} u_{2} \quad \text { in } \Omega_{2}, \quad \text { while } \quad u_{2} \leq u_{1}+a \text { on } \partial \Omega_{2} .
$$

Therefore, by the comparison principle $u_{2} \leq u_{1}+a$ in $\Omega_{2}$. In $\Omega_{1} \backslash \Omega_{2}, u_{2}=0$ and $u_{1}+a \geq 0$. We thus conclude

$$
u_{1}+a-u_{2} \geq 0 \quad \text { in } \Omega_{1} \cup \Omega_{2} .
$$

In particular, $u_{2} \leq a$ in $\Omega_{2} \backslash \Omega_{1}$ and $a>0$. Hence $\sup _{\Omega_{2} \backslash \Omega_{1}} u_{2} \leq a$.

We now claim

$$
\sup _{\Omega_{2} \backslash \Omega_{1}} u_{2}<a .
$$

Suppose on the contrary that there is a $z \in \Omega_{2} \backslash \Omega_{1}$ such that $u_{2}(z)=a$. Now, by (7), $u_{1}+a-u_{2} \geq 0$ in $\Omega_{2}$, and therefore it has a minimum value at $z$, and consequently a vanishing gradient. Since $z \in \Omega_{2} \backslash \Omega_{1}, u_{1}$ has also a vanishing gradient at $z$. Therefore we conclude that $\left|\nabla u_{2}\right|(z)=0$. By the convexity of $\Omega_{1} \cap \Omega_{2}$ we may take a ball $B$ in $\Omega_{2} \backslash \Omega_{1}$ with $z$ on its boundary and apply Lemma 2 to obtain a contradiction. This proves our claim. Summing up we have

$$
a=-u_{1}\left(y^{1}\right)>\sup _{\Omega_{2} \backslash \Omega_{1}} u_{2} \geq \frac{1}{q} d_{2}^{q} \geq \frac{1}{q} d_{1}^{q} .
$$

This proves the first part of the lemma. To prove the second statement observe by (7) that $u_{1}+a-u_{2}$ is nonnegative in $\Omega_{1}$. Also, by the definition of $a$ and since $y^{1} \notin \Omega_{2}$ we may conclude that $u_{1}+a-u_{2}$ has a minimum value at $y^{1}$ and thus $\nabla u_{1}\left(y^{1}\right)=\nabla u_{2}\left(y^{1}\right)=0$.

Remark. We remark that if $\inf \left(d_{1}, d_{2}\right)=0$, then $\Omega_{1} \equiv \Omega_{2}$, and consequently $u_{1} \equiv$ $u_{2}$. This depends on the fact that $\Omega_{1}$ and $\Omega_{2}$ have the same volume. The latter may be shown as follows. Since

$$
\left|\Omega_{1}\right|-\left|\Omega_{2}\right|=\int\left(\Delta_{p} u_{1}-\Delta_{p} u_{2}\right),
$$

we may integrate by parts, and use the fact that $\left|\nabla u_{j}\right|=0(j=1,2)$ outside a large ball, to conclude that $\left|\Omega_{1}\right|=\left|\Omega_{2}\right|$. 
Proof of Theorem 1. By the above remark if $\inf \left(d_{1}, d_{2}\right)=0$, then the theorem follows. Therefore suppose $0<d_{1} \leq d_{2}$. By Lemma 5 , there is a point $y^{1} \in \Omega_{1} \backslash \Omega_{2}$ such that

$$
a=-\inf _{\Omega_{1} \backslash \Omega_{2}} u_{1}=-u_{1}\left(y^{1}\right)>\frac{1}{q} d_{1}^{q}, \quad\left|\nabla u_{1}\left(y^{1}\right)\right|=0 .
$$

Let $H$ be the hyperplane passing through $y^{1}$ and containing $\Omega_{1} \cap \Omega_{2}$ on one side of it. By translation and rotation, we may assume that $y^{1}=0, H=\left\{x_{1}=0\right\}$ and $\Omega_{1} \cap \Omega_{2}$ lies in $\left\{x_{1}<0\right\}$. Now, choose $z \in \partial \Omega_{1} \backslash \Omega_{2}$ with largest distance to $H$. Then $z_{1} \leq d_{1}$ and by $(8), z_{1}^{q} / q<a$. Let $\epsilon>0$ be such that $\left(z_{1}+\epsilon\right)^{q} / q \leq a$, and define $w(x)=u_{1}(x)+a, v(x)=\left(\left(x_{1}+\epsilon\right)^{q}-\epsilon^{q}\right) / q$. Then, since $\Delta_{p} w=\Delta_{p} v=1$ in $D:=\left\{x \in \Omega_{1}: x_{1}>0\right\}$ and $w \geq v$ on $\partial D$, we'll have (by comparison principle) $w \geq v$ in $D$. Since also $w(0)=v(0)$, it follows that

$$
\frac{\partial w}{\partial x_{1}}(0) \geq \frac{\partial v}{\partial x_{1}}(0)
$$

i.e.

$$
\frac{\partial u_{1}}{\partial x_{1}}(0) \geq \frac{\partial v}{\partial x_{1}}(0)=\epsilon^{q-1},
$$

which contradicts (8). This proves the theorem in the case $d_{1} \leq d_{2}$. If $d_{2} \leq d_{1}$ we interchange $\Omega_{1}, \Omega_{2}$ and repeat the same argument. The proof is now completed.

Remark. It follows from the proof of Lemma 5 that one may weaken the convexity assumption on $\Omega_{1} \cap \Omega_{2}$ to an exterior ball condition if one assumes one of the solutions $u_{1}, u_{2}$ is positive.

\section{ACKNOWLEDGEMENTS}

The second author was partially supported by Swedish Natural Science Research Council. He also thanks the Mathematics Department at Tehran University for their hospitality and support during this work. Thanks go to Professors J. Lewis and P. Benilan for useful discussions on the p-Laplacian. Both authors thank A. Samii for her help in tex-formating the original file of the manuscript.

\section{REFERENCES}

[gu B. Gustafsson, On quadrature domains and an inverse problem in potential theory, J. Analyse Math. vol 55, 1990, 172-216. MR 92c:31013

[g-s] B. Gustafsson, H. Shahgholian, Existence and geometric properties of solutions of a free boundary problem in potential theory, J. Reine Angew. Math. 473 (1996), 137-179. MR 97e:35205

[h-k-m] J. Heinonen, T. Kilpeläinen, O. Martio, Nonlinear potential theory of degenerate elliptic equations, Oxford University Press, Oxford, 1993. MR 94e:31003

[i] V. Isakov, Inverse source problems, Math. Surveys Monographs, vol 34, Amer. Math. Soc., Providence, RI, 1990. MR 92g:35230

[k-s] L. Karp, H. Shahgholian, Regularity of free boundaries, To appear.

[1] J.L. Lewis, Regularity of the derivatives of solutions to certain degenerate elliptic equations, Indiana Univ. Math. J. 32, 1983, pp. 849-858. MR 84m:35048

[n] P. S. Novikov, Sur le probléme inverse du potentiel, Dokl. Akad. Nauk SSSR, vol 18, 1938, 165-168.

[sa] M. Sakai, Quadrature domains, Lecture Notes in Math., vol. 934, Springer-Verlag, BerlinHeidelberg, 1982. MR 84h:41047

[shah] H. Shahgholian, Convexity and uniqueness in an inverse problem of potential theory, Proc. Amer. Math. Soc. vol. 116, nr 4, 1992, 1097-1100. MR 93b:31008 
[sh] H. S. Shapiro, The Schwarz Function and its Generalization to Higher Dimensions, Wiley, New York, University of Arkansas Lecture Notes, 1992. MR 93g:30059

[t] P. Tolksdorf, On the Dirichlet problem for quasilinear equations in domains with conical boundary points, Comm. Partial Diff. Eq., 8(7), 1983, pp. 773-817. MR 85g:35053

[z] L. Zalcman, Some inverse problems of potential theory, Contemp. Math. vol 63, Amer. Math. Soc., Providence, RI, 1987, 337-350. MR 88e:31012

Department of Mathematics, University of Tehran, P.O. Box 13145-1873, Tehran, IRAN

Department of Mathematics, The Royal Institute of Technology, 10044 Stockholm, SWEDEN

E-mail address: henriks@math.kth.se 